\title{
Estilo de vida de adultos com deficiência percebida em quilombos baianos, Nordeste brasileiro
}

\author{
Style of living of adults with disability in baian quilombos, Northeast Brazil \\ Estilo de vida de adultos con discapacidad percibida en quilombos Bahianos, Nordeste brasileño
}

Recebido: 22/10/2021 | Revisado: 30/10/2021 | Aceito: 10/11/2021 | Publicado: 14/11/2021

\author{
Ricardo Franklin de Freitas Mussi \\ ORCID: https://orcid.org/0000-0003-1515-9121 \\ Universidade do Estado da Bahia, Brasil \\ E-mail: rimussi@yahoo.com.br \\ Camila Fabiana Rossi Squarcini \\ ORCID: https://orcid.org/0000-0002-1605-4834 \\ Universidade Estadual de Santa Cruz, Brasil \\ E-mail: csquarcini@gmail.com \\ Jorge Lopes Cavalcante Neto \\ ORCID: https://orcid.org/0000-0002-8396-2410 \\ Universidade do Estado da Bahia, Brasi \\ E-mail: jorgelcneto@hotmail.com
}

\begin{abstract}
Resumo
Para analisar a associação entre fatores sociodemográficos e o estilo de vida em adultos quilombolas baianos com deficiência percebida, 517 entrevistas face-a-face foram consideradas. Foi realizada regressão logística binária para definir a associação entre o Estilo de vida e os fatores sociodemográficos. A escolaridade permaneceu associada a Nutrição, a renda à Atividade Física, o número de filhos com Comportamento Preventivo e Relacionamentos, e o sexo com o Estresse. Então, ter menos escolaridade aumenta a probabilidade para Nutrição negativa, ter renda mais alta aumenta a probabilidade para Atividade Física positiva. Ter mais filhos aumenta a probabilidade do Comportamento Preventivo e Relacionamentos positivos e ser homem para Estresse positivo.

Palavras-chave: Grupo com Ancestrais do Continente Africano; Pessoas com deficiência; Estilo de vida; Condições sociais.
\end{abstract}

\begin{abstract}
To analyze the association between socio-demographic factors and lifestyle in Salvadoran quilombola adults with disabilities, 517 face-to-face interviews were considered. Binary logistic regression was performed to define the association between Lifestyle and sociodemographic factors. Schooling remained associated with Nutrition, Income to Physical Activity, Number of Children with Preventive Behavior and Relationships, and Sex with Stress. Therefore, having less schooling increases the likelihood for negative nutrition, having higher income increases the likelihood for positive physical activity. Having more children increases the likelihood of Preventive Behavior and Positive Relationships and being man to Positive Stress.
\end{abstract}

Keywords: African Continental Ancestry Group; People with disability; Lifestyle; Social conditions.

\section{Resumen}

Para analizar la asociación entre factores sociodemográficos y estilo de vida en adultos quilombolas salvadoreños con discapacidad, se consideraron 517 entrevistas presenciales. Se realizó una regresión logística binaria para definir la asociación entre el estilo de vida y los factores sociodemográficos. La escolaridad permaneció asociada con la nutrición, los ingresos por actividad física, el número de niños con conductas y relaciones preventivas y el sexo con estrés. Por lo tanto, tener menos escolaridad aumenta la probabilidad de una nutrición negativa, tener ingresos más altos aumenta la probabilidad de una actividad física positiva. Tener más hijos aumenta la probabilidad de tener un comportamiento preventivo y relaciones positivas y ser hombre frente al estrés positivo.

Palabras clave: Grupo de ascendencia continental africana; Personas con discapacidad; Estilo de vida; Condiciones sociales.

\section{Introdução}

As deficiências resultam da perda ou anormalidade estrutural ou fisiológica do corpo (Organização Mundial de Saúde, 1997) com algum grau de limitação e/ou impedimento físico, sensorial, mental e/ou intelectual para desempenho das atividades cotidianas. 
O último Censo Demográfico brasileiro realizado no ano de 2010 indicou 23,9\% da população brasileira com alguma deficiência, mais prevalente na região Nordeste $(26,3 \%)$ e, em pessoas negras $(27,1 \%)$ e amarelas $(27,1 \%)$ (Brasil, 2012). No entanto, a Pesquisa Nacional de Saúde de 2013 aponta que 6,2\% autorreferiram a presença de alguma deficiência (Malta et al, 2016). Contudo, essas prevalências podem ser inexatas, visto que as pessoas podem ter dificuldade em reconhecer a presença da deficiência e, que pessoas com deficiência, por vezes, sequer saem de casa, frequentam escolas ou são cadastradas no sistema público de saúde.

Embora a inclusão e participação social das pessoas com deficiência sejam ressaltadas nas discussões acadêmicas (Vasconcellos, Sena, 2021; Dawes, Coutinho, 2021; Franco, Oliveira Neto, 2020; Oliveira Neto, Melo, 2020; Carvalho, Cavalcanti, 2020) e, um pouco menos, em análises relativas à componentes do seu estilo de vida (Pereira, Freitas, 2021; Gomes et al, 2020), os serviços de saúde não desempenham papel satisfatório, estrutural e de serviços, para atendimento das demandas desta população (Rebouças et al., 2011), principalmente diante da ausência de informações populacionais e capacitação profissional.

Ao avaliar ou intervir com pessoas com deficiência é preciso considerar suas funcionalidades e não suas limitações e incapacidades (Farias; Buchalla, 2005; Souza Júnior; Correia; Queiroz, 2021). Os profissionais e serviços de saúde devem compreender os domínios da Classificação Internacional de Funcionalidade e Saúde (ICIF): funções e estruturas do corpo, atividades e participação e, fatores ambientais (Nordenfelt, 2003). A funcionalidade deve ser a condição de saúde prioritária, considerando o contexto ambiental que essas pessoas estão inseridas.

As doenças crônicas não transmissíveis (DCNT) emergem de uma relação multicausal complexa a partir da interação de elementos socioeconômicos, culturais e ambientais reconhecidos como fatores de risco comportamentais e fatores não modificáveis (Organização Pan-Americana da Saúde, 2009). O reconhecimento dos principais fatores sociodemográficos, econômicos e comportamentais associados à ocorrência e distribuição das enfermidades e fatores de risco populacional subsidiam o planejamento de ações de saúde mais específicos (Nogueira et al., 2016).

Aceitando o estilo de vida como importante elemento relacionado à saúde e qualidade de vida (Almeida, Casotti, Sena, 2018), investigações epidemiológicas sobre o comportamento e hábitos das populações fornecem dados para predições de risco à saúde (Danaei et al., 2009). Informações fundamentais para a promoção e proteção da saúde e, para o desenvolvimento de intervenções públicas com menor custo e maior eficiência (Green et al., 2005).

Dados internacionais indicam que pessoas com deficiências têm maior probabilidade para o estilo de vida negativo e desenvolvimento de DCNT (Clarke; Latham, 2014). No entanto, o Brasil ainda carece de informações sobre os hábitos desta população.

Estudo de base nacional brasileiro identificou importante associação de componentes do estilo de vida, eminentemente negativos, associados a população negra (Malta et al., 2015), exigindo atenção dos profissionais e serviços de saúde. Destarte, análises com a participação de populações afrodescendentes precisam considerar seus processos sóciohistóricos e particulares condições de vida (Almeida, Casotti, 2020; Mussi et al., 2020).

Parte importante da população negra brasileira reside em quilombos contemporâneos, comunidades autoatribuídas, vinculadas à terra, de ancestralidade negra e resistente à opressão histórica (Brasil, 2015; Mussi et al, 2020), com infraestrutura e acesso aos serviços públicos escassos (Almeida et al, 2019; Pereira, Mussi, 2020; Silva, Mussi, Rocha, 2020; Gomes et al., 2013), geralmente localizadas em espaços rurais, o que dificulta a prevenção do adoecimento e promoção da saúde.

Apesar de poucos, os estudos com quilombolas têm apontado a ampla presença de estilo de vida negativo na população adulta (Bezerra et al., 2015; Almeida et al, 2020; Rodrigues et al, 2020a), no entanto, nenhum com enfoque na presença de deficiência, seja como variável ou categoria de análise. 
Considerando as restritas informações sobre as pessoas com deficiência, menos ainda em quilombolas, que poderiam contribuir na avaliação e planejamento de ações voltadas para a saúde, este texto pretende analisar as associações entre fatores sociodemográficos e o estilo de vida de adultos quilombolas baianos com deficiência.

\section{Metodologia}

Esta análise é parte do estudo transversal intitulado "Perfil Epidemiológico dos Quilombolas baianos" autorizado pelo Comitê de Ética em Pesquisa com Seres Humanos da Universidade do Estado da Bahia (CEP/UNEB), parecer 1.386.019/2016, desenvolvido entre os meses de fevereiro a novembro de 2016.

A microrregião geoeconômica de Guanambi/Bahia, com 42 quilombos certificados pela Fundação Palmares (2016) no ano de 2016, distribuídos em 10 municípios, foi o campo empírico. A população foi determinada considerando a presença de 80 famílias por quilombo (Brasil, 2015), com dois adultos ( $\geq 18$ anos) por família, em cada um dos quilombos, totalizando 6720 adultos.

O cálculo amostral adotou prevalência desconhecida para o desfecho, nível de confiança de $95 \%$, erro amostral de 5 pontos percentuais, efeito do delineamento de 1,5 (quilombo como conglomerado), acréscimo de $30 \%$ para recusas e $20 \%$ para perdas e confundimento, determinando amostra mínima de 818 sujeitos. No entanto, participaram 1025 quilombolas com $17,07 \%$ de recusas, definindo amostra final de 850 participantes.

A amostra foi obtida pelo sorteio de 17 quilombos (incluindo três recusas), seguido de contato para apresentação da proposta e agendamentos das visitações para realização das coletas. Todos os adultos residentes nos quilombos durante as coletas foram considerados elegíveis. Aqueles sem condição de raciocínio ou comunicação independente foram excluídos das entrevistas.

Durante as visitações para coleta de dados foram realizadas entrevistas face-a-face desenvolvidas por equipe capacitada e legalmente habilitada para a função.

Foi considerado com deficiência aqueles que responderam 'leve', 'médio(a)', 'intenso(a)' e 'não consegue' para as perguntas: 1) Em geral, que grau de dificuldade o(a) Sr.(a) tem para se locomover? 2) Em geral, que grau de dificuldade o(a) Sr.(a) tem para ouvir? 3) Em geral, que grau de dificuldade o(a) Sr.(a) tem para ver de longe? E para ver de perto? Conforme questionário da Pesquisa Nacional de Saúde, convalidado para população quilombola (Bezerra et al., 2014).

O estilo de vida foi investigado considerando as dimensões do questionário de Perfil do Estilo de Vida Individual (nutrição, atividade física, comportamento preventivo, relacionamentos, controle do stress), validado para adultos brasileiros (Both et al., 2008).

Cada dimensão é composta por três perguntas que possibilitam quatro respostas (não, às vezes, quase sempre, sempre) com pontuação progressiva de zero à três pontos. O somatório das respostas, por dimensão, foi categorizado arbitrariamente em negativo (0-4 pontos) e positivo (5-9 pontos).

As variáveis sociodemográficas foram: sexo (feminino, masculino); grupo etário ( $<40$ anos, $>40$ anos); raça/cor da pele (outras, negra); situação conjugal (com companheiro, sem companheiro); escolaridade ( $>4$ anos, $\leq 4$ anos); situação laboral

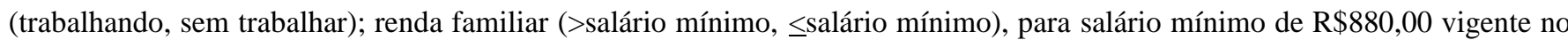
ano de 2016: quantidade de filhos ( $\leq 4$ filhos, $>4$ filhos); residentes no lar ( $\leq 4$ pessoas, $>4$ pessoas).

Realizou-se análise de associação bivariada por meio do quiquadrado, com nível de significância de 5\% e cálculo da razão de odds (Odds Ratio=OR) com Intervalo de Confiança (IC) de 95\%. As variáveis com p-valor $\leq 0,20$ na análise bivariada foram incluídas em modelos de Regressão Logística binária, do tipo Stepwise, considerando modelos individuais para cada dimensão do estilo de vida. Todas as análises foram realizadas no Statistical Package for the Social Sciences (SPSS), versão 20.0 . 


\section{Resultados}

Dos 850 participantes da coleta original, 517 (62,4\%) referiram alguma deficiência (física, auditiva e/ou visual), compondo a amostra desta análise. Destes, 61,7\% (319) eram do sexo feminino, 77,8\% (402) tinham mais de 40 anos, 85,1\% (440) autorreferidos negros, 79,7\% (412) com relacionamento conjugal, 63,6\% (329) até 4 anos de estudo, 51,1\% (264) com trabalho remunerado, 66,0\% (341) com renda familiar inferior ao salário mínimo, 65,0\% (336) com até 4 filhos e 66,3\% (343) com até quatro moradores no lar.

Quanto as dimensões do estilo de vida, 52,0\% (269) apresentaram nutrição positiva, 59,2\% (306) atividade física negativa, $73,3 \%$ (379) comportamento preventivo positivo, 91,7\% (474) relacionamentos positivos e 76,4\% (395) com controle do estresse positivo.

A análise de associação bivariada entre a Nutrição e fatores sociodemográficos (tabela 1) indica que a escolaridade $(\mathrm{OR}=1,70$; IC95\%: 1,14:2,53) estive significativamente associada a essa dimensão. Neste sentido, os sujeitos com maior escolaridade apresentam Nutrição mais positiva quando comparado àqueles com menor escolaridade, respectivamente.

A análise bivariada entre o estilo de vida na dimensão Atividade Física e os fatores sociodemográficos (Tabela 1) mostrou associação entre Trabalho (OR=0,66; IC95\%: 0,46:0,95) e Renda Familiar (OR=0,48; IC95\%: 0,32:0,75). Este comportamento positivo foi significativamente mais presente entre os sujeitos que possuíam trabalho remunerado e com renda familiar mensal >1 salário mínimo, quando comparados, respectivamente, aos sujeitos sem trabalho remunerado e aqueles com renda familiar mensal $\leq 1$ salário mínimo.

Tabela 1. Análise bivariada da associação entre o estilo de vida pelas dimensões 'Nutrição' e 'Atividade Física' com os fatores sociodemográficos ( $\mathrm{n}=517)$. Bahia, Brasil, 2016.

\begin{tabular}{|c|c|c|c|c|c|c|c|c|}
\hline \multirow[b]{2}{*}{ Variáveis sociodemográficas } & \multicolumn{4}{|c|}{ NUTRIÇÃO } & \multicolumn{4}{|c|}{ ATIVIDADE FÍSICA } \\
\hline & $\mathbf{n}$ & $\begin{array}{c}\% \\
\text { negativa }\end{array}$ & OR (IC95\%) & $\begin{array}{c}\text { p- } \\
\text { valor }\end{array}$ & $\mathbf{n}$ & $\begin{array}{c}\% \\
\text { negativa }\end{array}$ & OR (IC95\%) & $\begin{array}{c}\text { p- } \\
\text { valor }\end{array}$ \\
\hline \multicolumn{9}{|l|}{ Sexo } \\
\hline Feminino & 315 & 44,4 & $1,343(0,939: 1,922)$ & 0,107 & 315 & 62,2 & $0,795(0,552: 1,144)$ & 0,217 \\
\hline Masculino & 195 & 51,8 & & & 194 & 56,7 & & \\
\hline \multicolumn{9}{|l|}{ Grupo etário } \\
\hline$<40$ anos & 114 & 47,4 & $0,994(0,655: 1,509)$ & 0,978 & 112 & 61,6 & $0,923(0,600: 1,420)$ & 0,716 \\
\hline$\geq 40$ anos & 396 & 47,2 & & & 397 & 59,7 & & \\
\hline \multicolumn{9}{|l|}{ Raça-cor da pele } \\
\hline Outras & 74 & 45,9 & $1,063(0,649: 1,743)$ & 0,807 & 74 & 60,8 & $0,967(0,584: 1,601)$ & 0,895 \\
\hline Negra & 436 & 47,5 & & & 435 & 60,0 & & \\
\hline \multicolumn{9}{|l|}{ Situação Conjugal } \\
\hline Com companheiro & 408 & 48,5 & $1,294(0,835: 2,005)$ & 0,250 & 407 & 61,2 & $1,244(0,802: 1,929)$ & 0,329 \\
\hline Sem companheiro & 102 & 42,2 & & & 102 & 55,9 & & \\
\hline \multicolumn{9}{|l|}{ Escolaridade } \\
\hline$>4^{\mathrm{a}}$ série & 145 & 38,6 & $1,701(1,141: 2,536)$ & 0,009 & 145 & 55,9 & $1,213(0,816: 1,804)$ & 0,340 \\
\hline$\leq 4^{\mathrm{a}}$ série & 323 & 51,7 & & & 322 & 60,6 & & \\
\hline \multicolumn{9}{|l|}{ Situação Laboral } \\
\hline Trabalhando & 262 & 46,9 & $0,975(0,688: 1,380)$ & 0,886 & 262 & 55,3 & $0,662(0,463: 0,947)$ & 0,024 \\
\hline Sem trabalhar & 248 & 47,6 & & & 247 & 65,2 & & \\
\hline \multicolumn{9}{|l|}{ Renda Familiar } \\
\hline$>1$ salário mínimo & 113 & 43,4 & $0,842(0,548: 1,293)$ & 0,431 & 113 & 47,8 & $0,487(0,316: 0,750)$ & 0,001 \\
\hline$\leq 1$ salário mínimo & 338 & 47,6 & & & 337 & 65,3 & & \\
\hline \multicolumn{9}{|l|}{ Número de filhos } \\
\hline$\leq 4$ filhos & 333 & 45,9 & $1,204(0,832: 1,744)$ & 0,324 & 332 & 60,5 & $0,931(0,639: 1,357)$ & 0,710 \\
\hline$>4$ filhos & 170 & 50,6 & & & 170 & 58,8 & & \\
\hline \multicolumn{9}{|l|}{ Moradores no lar } \\
\hline$\leq 4$ pessoas & 339 & 46,9 & $1,055(0,730: 1,525)$ & 0,770 & 339 & 61,7 & $0,838(0,576: 1,220)$ & 0,356 \\
\hline$>4$ pessoas & 170 & 48,2 & & & 169 & 57,4 & & \\
\hline
\end{tabular}

Fonte: Autores. 
A Tabela 2 apresenta a análise de associação bivariada entre o estilo de vida tabela 2 apresenta a análise de associação bivariada entre o estilo de vida pela dimensão Comportamento Preventivo e fatores sociodemográficos. Situação Conjugal (OR=1,96; IC95\%: 1,11:3,44), Número de filhos (OR=0,59; IC95\%: 0,38:0,92) e Número de residentes no lar (OR=1,87; IC95\%: 1,24:2,82) se associaram ao Comportamento Preventivo. Não ter companheiro(a), $>4$ filhos e $\leq 4$ residentes no lar foram associados significativamente com o Comportamento Preventivo positivo.

Ainda na Tabela 2, são apresentados os dados da associação bivariada entre o estilo de vida pela categoria Relacionamentos e fatores sociodemográficos. Neste caso, ocorreu associação com a situação conjugal (OR=0,45; IC95\%: 0,22:0,94), evidenciando que a maioria daqueles com companheiro(a) apresentaram Relacionamentos positivo.

Tabela 2. Análise bivariada da associação entre o estilo de vida pelas dimensões 'Comportamento Preventivo' e 'Relacionamentos' com os fatores sociodemográficos (n=517). Bahia, Brasil, 2016.

\begin{tabular}{|c|c|c|c|c|c|c|c|c|}
\hline \multirow[b]{2}{*}{$\begin{array}{l}\text { Variáveis } \\
\text { sociodemográficas }\end{array}$} & \multicolumn{4}{|c|}{ COMPORTAMENTO PREVENTIVO } & \multicolumn{4}{|c|}{ RELACIONAMENTOS } \\
\hline & $\mathbf{n}$ & $\begin{array}{c}\% \\
\text { negativo }\end{array}$ & OR (IC95\%) & $\begin{array}{c}\text { p- } \\
\text { valor }\end{array}$ & $\mathbf{n}$ & $\begin{array}{c}\% \\
\text { negativo }\end{array}$ & OR (IC95\%) & $\begin{array}{c}\text { p- } \\
\text { valor }\end{array}$ \\
\hline \multicolumn{9}{|l|}{ Sexo } \\
\hline Feminino & 316 & 25,0 & 1,120 & 0,585 & 315 & 7,9 & 0,630 & 0,232 \\
\hline Masculino & 195 & 27,2 & $(0,747: 1,680)$ & & 194 & 5,2 & $(0,296: 1,343)$ & \\
\hline \multicolumn{9}{|l|}{ Grupo etário } \\
\hline$<40$ anos & 114 & 30,7 & 0,730 & 0,179 & 114 & 8,8 & 0,703 & 0,366 \\
\hline$\geq 40$ anos & 397 & 24,4 & $(0,461: 1,155)$ & & 395 & 6,3 & $(0,327: 1,510)$ & \\
\hline \multicolumn{9}{|l|}{$\overline{\mathbf{R}}$ aça-cor da pele } \\
\hline Outras & 74 & 31,1 & 0,737 & 0,266 & 74 & 8,1 & 0,810 & 0,651 \\
\hline Negra & 437 & 24,9 & $(0,430: 1,262)$ & & 435 & 6,7 & $(0,324: 2,023)$ & \\
\hline \multicolumn{9}{|l|}{ Situação Conjugal } \\
\hline Com companheiro & 409 & 28,1 & 1,956 & 0,020 & 407 & 5,7 & 0,449 & 0,033 \\
\hline Sem companheiro & 102 & 16,7 & $(1,113: 3,436)$ & & 102 & 11,8 & $(0,215: 0,937)$ & \\
\hline \multicolumn{9}{|l|}{ Escolaridade } \\
\hline$>4^{\mathrm{a}}$ série & 145 & 22,8 & 1,076 & 0,757 & 145 & 6,9 & 0,942 & 0,880 \\
\hline$\leq 4^{\mathrm{a}}$ série & 324 & 24,1 & $(0,676: 1,712)$ & & 322 & 6,5 & $(0,432: 2,054)$ & \\
\hline \multicolumn{9}{|l|}{ Situação Laboral } \\
\hline Trabalhando & 263 & 25,5 & 0,962 & 0,850 & 263 & 4,9 & 0,529 & 0,079 \\
\hline Sem trabalhar & 248 & 26,2 & $(0,648: 1,430)$ & & 246 & 8,9 & $(0,261: 1,076)$ & \\
\hline \multicolumn{9}{|l|}{ Renda Familiar } \\
\hline >1 salário mínimo & 113 & 19,5 & 0,595 & 0,051 & 113 & 4,4 & 0,532 & 0,200 \\
\hline <1salário mínimo & 339 & 28,9 & $(0,353: 1,002)$ & & 337 & 8,0 & $(0,200: 1,415)$ & \\
\hline \multicolumn{9}{|l|}{ Número de filhos } \\
\hline$\leq 4$ filhos & 333 & 28,8 & 0,590 & 0,021 & 331 & 8,5 & 0,462 & 0,075 \\
\hline$>4$ filhos & 171 & 19,3 & $(0,377: 0,924)$ & & 171 & 4,1 & $(0,197: 1,080)$ & \\
\hline \multicolumn{9}{|l|}{ Moradores no lar } \\
\hline$\leq 4$ pessoas & 339 & 21,5 & & & 338 & 6,2 & & \\
\hline$>4$ pessoas & 171 & 33,9 & $\begin{array}{c}1,870 \\
(1,242: 2,815) \\
\end{array}$ & 0,003 & 170 & 8,2 & $\begin{array}{c}1,355 \\
(0,671: 2,736) \\
\end{array}$ & 0,397 \\
\hline
\end{tabular}

Fonte: Autores.

Na Tabela 3 estão apresentadas as análises de associações entre o estilo de vida pela dimensão Controle do Estresse e fatores sociodemográficos. Apenas o sexo esteve associado significativamente (OR=0,64; IC95\%: 0,41:0,99) com o Controle do Estresse. O que evidencia que a maioria dos indivíduos do sexo masculino apresentou a categoria Controle do Estresse positivo, quando comparados aos indivíduos do sexo feminino. 
Tabela 3. Análise bivariada da associação entre o estilo de vida pela dimensão Controle do Estresse e fatores sociodemográficos $(\mathrm{n}=517)$. Bahia, Brasil, 2016.

\begin{tabular}{|c|c|c|c|c|}
\hline Variáveis sociodemográficas & $\mathbf{n}$ & \% negativo & OR (IC95\%) & p-valor \\
\hline \multicolumn{5}{|l|}{ Sexo } \\
\hline Feminino & 317 & 25,6 & $0,635(0,407: 0,990)$ & 0,045 \\
\hline Masculino & 195 & 17,9 & & \\
\hline \multicolumn{5}{|l|}{ Grupo etário } \\
\hline$<40$ anos & 114 & 27,2 & $0,729(0,453: 1,175)$ & 0,195 \\
\hline$\geq 40$ anos & 397 & 21,4 & & \\
\hline \multicolumn{5}{|l|}{$\overline{\mathbf{R}}$ aça-cor da pele } \\
\hline Outras & 74 & 16,2 & $1,614(0,837: 3,110)$ & 0,153 \\
\hline Negra & 437 & 23,8 & & \\
\hline \multicolumn{5}{|l|}{ Situação Conjugal } \\
\hline Com companheiro & 409 & 22,5 & $0,943(0,565: 1,575)$ & 0,823 \\
\hline Sem companheiro & 102 & 23,5 & & \\
\hline \multicolumn{5}{|l|}{ Escolaridade } \\
\hline$>4^{\mathrm{a}}$ série & 145 & 23,4 & $0,883(0,554: 1,410)$ & 0,603 \\
\hline$\leq 4^{\mathrm{a}}$ série & 324 & 21,3 & & \\
\hline \multicolumn{5}{|l|}{ Situação Laboral } \\
\hline Trabalhando & 263 & 20,5 & $0,775(0,512: 1,174)$ & 0,229 \\
\hline Sem trabalhar & 248 & 25,0 & & \\
\hline \multicolumn{5}{|l|}{ Renda Familiar } \\
\hline >1 salário mínimo & 113 & 19,5 & $0,783(0,461: 1,328)$ & 0,364 \\
\hline$\leq 1$ salário mínimo & 339 & 23,6 & & \\
\hline \multicolumn{5}{|l|}{ Número de filhos } \\
\hline$\leq 4$ filhos & 333 & 23,4 & $0,903(0,579: 1,407)$ & 0,651 \\
\hline$>4$ filhos & 171 & 21,6 & & \\
\hline \multicolumn{5}{|l|}{ Moradores no lar } \\
\hline$\leq 4$ pessoas & 339 & 20,6 & $1,414(0,922: 2,170)$ & 0,113 \\
\hline$>4$ pessoas & 171 & 26,9 & & \\
\hline
\end{tabular}

Fonte: Autores.

A Tabela 4 apresenta os modelos finais de regressão logística para cada categoria de estilo de vida e variáveis sociodemográficas remanescentes. Observou-se que a escolaridade permaneceu associada com as categorias Nutrição, a renda familiar com Atividade Física, número de filhos com Comportamento Preventivo e Relacionamentos e, sexo com controle do estresse. Contudo, esses modelos de Regressão Logística foram fracos para explicar as associações aqui requeridas, uma vez que o os valores do R-Square foram $\leq 0,08$. Ou seja, a variável dependente (Estilo de Vida) em suas categorias (Nutrição, Atividade Física, Comportamento Preventivo, Relacionamento e Estresse) pode ser explicada apenas em até $0,8 \%$ pelas variáveis sociodemográficas Escolaridade, Renda Familiar, Número de filhos, Sexo, respectivamente para cada categoria.

Tabela 4. Modelo final de Regressão Logística binária sobre fatores associados para cada categoria de estilo de vida remanescente.

\begin{tabular}{|c|c|c|c|c|c|c|}
\hline $\begin{array}{l}\text { Likelihood de fatores associados } \\
\text { Modelo final }\end{array}$ & B & $\operatorname{Exp}(\mathbf{B})$ & P-valor & Constant & $-2 \log$ & R-Square \\
\hline Escolaridade*Nutrição & $-0,531$ & 0,588 & 0,009 & 0,068 & 640,835 & 0,02 \\
\hline Renda Familiar*Atividade Física & $-0,720$ & 2,054 & 0,01 & $-0,089$ & 591,621 & 0,03 \\
\hline $\begin{array}{l}\text { Número de filhos*Comportamento } \\
\text { Preventivo }\end{array}$ & 0,971 & 2,641 & $<0,001$ & $-1,057$ & 489,058 & 0,08 \\
\hline Número de filhos*Relacionamento & 0,909 & 2,481 & 0,04 & $-3,611$ & 225,495 & 0,02 \\
\hline Sexo*Estresse & 0,448 & 1,566 & 0,04 & $-1,514$ & 542,866 & 0,01 \\
\hline
\end{tabular}




\section{Discussão}

Possivelmente este seja o primeiro estudo que investiga o perfil do estilo de vida, considerando as categorias nutrição, $\mathrm{AF}$, comportamento preventivo, relacionamentos e controle do estresse, em adultos quilombolas com deficiência. Destarte, esta investigação detectou a recorrência do baixo consumo de vegetais in natura, da alta ingestão de alimentos gordurosos e açucarados, além da realização inadequada da quantidade de refeições diárias, promovendo comportamento nutricional negativo em metade dos participantes, principalmente entre os homens.

Neste sentido, inquérito populacional brasileiro identificou que ser homem se associa com hábitos alimentares negativos, como o consumo de alimentos gordurosos e refrigerantes (Claro et al., 2015). No entanto, também foram identificadas associações com escolaridade, grupos etários mais jovens e raça-cor negra, para estes grupos alimentares. Outra análise com amostra representativa brasileira apontou que aproximadamente 1/3 dos adultos consomem cinco ou mais porções de vegetais ao dia (Claro et al., 2015), principalmente os mais velhos e com melhor escolaridade. Enquanto pessoas negras apresentaram os menores índices de frequência alimentar de frutas e hortaliças.

Diante da possibilidade da elevada inadequação na quantidade de refeições desenvolvida pelos participantes desta análise com quilombolas, é importante ressaltar que a realização de menos que três refeições por dia se associou com o consumo de alimentos menos saudáveis e a presença de fatores de risco metabólicos (Holmbäck et al., 2010).

O comportamento nutricional inadequado observado eminentemente entre os quilombolas com deficiência com baixa escolaridade pode ser explicado similarmente ao que se observa na população em geral, considerando que os sujeitos moldam seus comportamentos pela observação e convivência. As pessoas costumam comer o que seus grupos sociais comem e os hábitos familiares são essenciais na determinação do perfil alimentar individual (Quaioti; Almeida, 2006). No entanto, também é importante considerar, a já relatada dificuldade para aquisição de alimentos mais saudáveis por quilombolas (Bezerra et al., 2013) impactando negativamente em seu comportamento nutricional.

Dada a importância de uma educação nutricional (Bonsmann; Wills, 2012), pessoas que apresentam maior escolaridade tendem a procurar maiores informações acerca de uma alimentação saudável e balanceada, o que as tornam mais suscetíveis a consumir alimentos que sejam benéficos à saúde, como frutas e hortaliças em detrimento ao consumo de frituras e alimentos industrializados.

Apesar do trabalho de muitos adultos quilombolas ser baseado na agricultura de subsistência (Oliveira et al, 2021), produzindo os alimentos para o consumo das famílias e da comunidade, este estudo apontou que o contexto alimentar e nutricional dos quilombolas com deficiência ainda está aquém do ideal para um comportamento nutricional adequado, especificamente dentre aqueles com menor escolaridade. Isso coaduna com o perfil de pessoas com deficiência (Weil et al., 2002), que em sua maioria apresenta condições sociodemográficas desfavoráveis, como baixa escolaridade, que os colocam numa situação de limitação social e pessoal, imposta pela própria condição de deficiência.

No caso do comportamento nutricional, muitas pessoas com deficiência acabam por não terem uma alimentação adequada devido a incapacidade ou dificuldade em conseguir se deslocar para comprar ou preparar alimentos, manusear utensílios de cozinha ou fogão (Hall et al., 2003; Tomey et al., 2005). Tais questões aliadas ao fato dessas pessoas terem mais baixa escolaridade as coloca em risco importante ao comportamento alimentar e nutricional inadequado.

A prática regular de AF contribui na prevenção do desenvolvimento de DCNT e seus agravos (Organização Mundial de Saúde, 2010). De maneira mais específica, a revisão de literatura identificou que níveis ideais de AF em populações afrodescendentes diminuem a carga alostática entre mulheres, a fragilidade óssea entre idosos e o risco de morte, aumentando a concentração de vitamina D (Almeida, Casotti, 2020). Apesar dos relatos da influência negativa da deficiência para o 
desenvolvimento das AF (Lehnhard et al., 2012), é importante ressaltar que os benefícios da sua prática também podem, e devem, ser usufruídos por todas as pessoas, inclusive aquelas com deficiência.

Esta investigação com adultos quilombolas com deficiência identificou elevada prevalência do estilo de vida pouco ativo, analisado a partir da prática de $\mathrm{AF}$ de tempo livre e de deslocamento, eminentemente entre aqueles com menor renda. Neste sentido, já foi identificado que 22,5\% e 31,9\% dos adultos brasileiros atingem a recomendação de quantidade de $\mathrm{AF}$ praticada no tempo livre e no deslocamento respectivamente (Mielke et al., 2015). Portanto, a condição identificada nesta análise quilombola corrobora o indicativo que a maioria absoluta da população encontra-se em situação de risco para o adoecimento e obesidade diante da recorrência do estilo de vida pouco ativo fisicamente.

No mesmo sentido, em população quilombola de município do sudoeste baiano foi relatado que $13,1 \%$ dos adultos atingiram os 150 minutos de AF no tempo livre (sexo masculino, menor faixa etária e maior escolaridade) (Bezerra et al., 2015). Enquanto em comunidade do Alto Sertão baiano foi verificado que 52,7\%, principalmente mulheres e pessoas mais velhas, não praticavam nenhum tipo de $\mathrm{AF}$ em seu tempo livre (Mussi et al., 2015). Já considerando a AF total, investigação regional no sertão baiano identificou que $21,9 \%$ dos adultos quilombolas não atingiam os níveis ideais de prática (Rodrigues et al, 2020a).

Assim, fica evidenciado que parece haver uma recorrência da baixa adesão ao comportamento fisicamente ativo, recomendado para a obtenção e/ou manutenção da saúde, em adultos quilombolas com e/ou sem deficiência. Neste contexto, a falta de prontidão para a prática de AF, ou seja, a manifestação/presença de alguma contraindicação para a adoção de estilo de vida ativo nesta população emerge como elemento gerador de barreira/impedimento para sua adoção, como já foi identificado em 62,9\% dos quilombolas participantes de análise regional (Rodrigues et al, 2020b).

Os comportamentos preventivos, abordados neste levantamento, constituem elementos pertencentes a prevenção primária (respeito às leis de trânsito e abstenção do consumo de drogas licitas) e secundária (rastreio precoce de DCNT) em saúde (Brasil, 2008), fundamentais na redução do risco de incapacidades temporárias ou persistentes em adultos. Cerca de 70\% dos quilombolas participantes apresentam menor probabilidade para o desenvolvimento de DCNT, vícios e lesões, principalmente entre aqueles com $>4$ filhos.

Este quadro resultaria da compreensão que possuir mais filhos demanda maiores responsabilidades e expectativas frente ao futuro da família e dos seus descendentes. Diante disso, aqueles sujeitos com maiores responsabilidades familiares, buscam se proteger de situações que envolvam risco de sofrerem violência e acidentes e, evitam o uso de drogas lícitas. O que poderia explicar a associação encontrada neste estudo.

O papel familiar tem importância fundamental no comportamento das pessoas, pois mesmo diante das mudanças ocorridas nos seus padrões, ter uma família estruturada figura como um importante fator de proteção contra o consumo de drogas (Schenker; Minayo, 2004). Mesmo sem ter investigado as organizações familiares em detalhes, supõe-se que no contexto quilombola essas pessoas com maior número de filhos possuem famílias mais estruturadas e consequentemente maior comportamento preventivo.

Diferentemente dos achados desta análise, investigação na população brasileira identificou o consumo de drogas lícitas associado com grupos etários, álcool com os mais jovens e fumo com os mais velhos (Malta et al., 2015). Ainda foi identificado que ser homem, negro e com menor instrução aumenta a probabilidade para o consumo de bebida alcoólica e o hábito tabagista.

A identificação dos grupos populacionais mais susceptíveis à dependência química é fundamental para o planejamento e execução de estratégias de combate e redução dos seus danos. Investigação com quilombolas determinou que o consumo de bebida alcoólica esteve associado aos grupos etários mais jovens, aos homens e, aqueles com maior envolvimento em acidentes e violência (Cardoso et al., 2015). 
É reconhecido que a manutenção de bons relacionamentos se associa com menores índices de morbimortalidade e com melhores indicativos de bem-estar geral. Neste sentido, os quilombolas com deficiência que possuíam filhos apresentam relacionamentos mais positivos, considerando a manutenção das amizades, participação em atividades sociais e atuação para a melhoria da comunidade.

Tendo como base que o círculo familiar, formado pelo(a) companheiro(a) e filhos comumente constituem as pessoas mais próximas do círculo de convivência dos indivíduos, sendo os filhos muitas vezes, na condição de deficiências dos pais, figuras centrais na rede de suporte social, suas presenças fortalecem as relações sociais de maneira geral, e no caso de pessoas com deficiência muitas vezes os filhos são decisivos para o compartilhamento de alegrias, angústias, inquietações e as mais diferentes emoções. Dada a dificuldade e desafio em manter uma família por parte da pessoa com deficiência (Llewellyn; Hindmarsh, 2015), infere-se que quando há mais filhos no lar, esses são capazes de reforçar o suporte social de seus pais, fortalecer seus relacionamentos, tornando-os mais positivos.

Ao considerar as peculiaridades do contexto quilombola, pode-se inferir que possuir mais filhos permite que as pessoas com deficiência dessas comunidades sejam mais socialmente inseridas nas atividades e grupos comunitários, o que as tornam mais funcionais e participativas. Esse fato é relevânte, pois um dos maiores impecilhos às pessoas com deficiência são os fracos vínculos de relacionamentos e participação social (Holanda et al., 2015).

Diante da importância que as redes de apoio/suporte social desempenham na saúde dos indivíduos, é preciso considerar a situação familiar, como o número de filhos de pessoas com deficiência, sobretudo àquelas de contextos específicos, como os quilombolas, em programas de atenção e proteção à saúde. Pois, parece que possuir mais filhos representa fator protetivo que pode e deve ser considerado no momento da elaboração de estratégias de intervenções em Saúde Pública.

As iniquidades e o racismo impactam negativamente na ampliação da presença de doenças crônicas das mais variadas naturezas, inclusive as mentais (Faro; Pereira, 2011; Mussi; Rocha; Alves, 2019). Então, ser negro elevaria a probabilidade da presença mais recorrente do estresse.

No entanto, estudo com quilombolas apontou prevalência de transtorno mental similar a encontrada na população brasileira, principalmente associado à prática insuficiente de $\mathrm{AF}$ e serviços de saúde deficitários (Barroso et al., 2014). Mas, o estudo alerta que a presença insuficiente ou mesmo ausência do serviço público de saúde pode ter gerado subnotificação de pessoas com esse grupo de doenças.

Na presente investigação, questões relacionadas ao descontrole dos níveis de estresse, como a reduzida presença de tempo para relaxar, a participação em debate com alteração de humor e o desequilíbrio entre tempo livre e laboral, foram mais presentes no sexo feminino. O que corrobora o indicativo que mulheres são mais sensíveis aos fatores estressantes (Calais $e t$ al., 2003) e possuem as mais altas prevalências de problemas de saúde mental (Yokota et al., 2016; Mussi; Rocha; Alves, 2019).

Sabe-se que o trabalho atua na construção da identidade, pelas relações cotidianas, e contribui na inserção do indivíduo no mundo, possibilitando reconhecimento social, promovendo autoestima e permitindo afirmação pessoal (Cruz et al., 2015). No entanto, o descompasso entre o tempo dedicado ao trabalho e a pouca disponibilidade de tempo livre para atividades recreativas e sociais pode acarretar o aumento dos níveis de estresse.

Reconhecendo que a saúde mental é condição indispensável ao bom funcionamento do organismo e das relações sociais (Kawachi; Berkman, 2001), mudanças de comportamento que venham a fortalecer a saúde mental dos quilombolas com deficiência, especificamente as mulheres, são fundamentais.

Contudo, a atenção à saúde mental de populações mais vulneráveis ainda não é prioridade dentro dos programas de saúde pública existentes no Brasil (Mateus et al., 2008). Enquanto essa questão não for item prioritário nas políticas nacionais, 
diversas pessoas continuarão sofrendo e adoecendo sem nem mesmo entender como lidar com a sintomatologia do problema psíquico, elevando seu sofrimento.

Cabe destacar que muitas mulheres quilombolas com deficiência apresentaram comportamento de risco frente ao estresse. Esse achado é um indicativo importante do impacto que tais condições podem ter na saúde mental dessa população. Devido à escassez de estudos e a pouca atenção dada às condições de saúde da população quilombola, pessoas com deficiência desse contexto poderão ser "falácias estatísticas" de um universo ainda desconhecido para muitos gestores no campo da saúde.

O quadro epidemiológico negativo relativo ao estilo de vida entre os adultos quilombolas com deficiência de região baiana preocupa, especialmente diante do reconhecimento da problemática relativa à presença, disponibilidade, acesso e utilização dos serviços (Almeida et al, 2019; Pereira, Mussi, 2020; Silva, Mussi, Rocha, 2020; Gomes et al., 2013), que atrapalham ações de promoção, de prevenção e de recuperação da saúde por esta população.

Dentre as limitações desta análise é reconhecida a incapacidade da determinação causal entre a presença da deficiência e os componentes do estilo de vida, característica indissociável dos estudos seccionais. Ao se utilizar a entrevista como instrumento de coleta de dados, deve ser considerado o viés de memória e compreensão do participante ao responderem as questões referentes à autodeterminação das deficiências e referente ao estilo de vida individual.

Outro fator a ser considerado como limitação é a possibilidade da adoção de categorização arbitrária dos resultados do estilo positivo e negativo, diferentemente de como foi validado (Both et al., 2008), justificada pela amostra relativamente reduzida deste estudo, especialmente entre os homens. Finalmente, deve-se considerar os resultados das análises com cuidado visto que seus resultados apresentaram associações frágeis, possivelmente devido a amostra pequena.

Além disso, devido os fracos modelos de regressão logística evidenciados no presente estudo, as associações encontradas precisam ser consideradas com cautela, pois variáveis sociodemográficas ainda não investigadas neste estudo poderiam explicar de forma mais incisiva comportamentos de estilos de vida entre a população quilombola.

\section{Conclusão}

Este estudo evidenciou que ter menor escolaridade é fator de risco para um comportamento nutricional positivo. Por outro lado, ter maior renda é fator de proteção para um estilo de vida mais ativo, possuir mais que 4 filhos é fator de proteção ao comportamento preventivo e de relacionamento positivos e ser do sexo masculino é fator de proteção para níveis mais baixos de estresse.

Estes achados contribuem na compreensão dos comportamentos de risco e protetivos que quilombolas com deficiência estão expostos. Estudo como este em população negra e rural contribuirá no embasamento e desenvolvimento de intervenções voltadas para a melhoria social e, portanto, da saúde nas comunidades quilombolas.

\section{Agradecimentos}

Agradecemos as Associações de Moradores das Comunidades Quilombolas participantes do estudo pela importante colaboração na logística local para viabilização das coletas de dados.

\section{Referências}

Almeida, C. B. \& Casotti, C. A. (2020). Estilo de vida da população afrodescendente: revisão integrativa. Journal Nursing Health, 10 (n.esp.), e20104012.

Almeida, C. B. \& Casotti, C. A. (2021). Revisão integrativa: comportamentos sedentários, síndrome metabólica e seus componentes em populações quilombolas. International Journal of Development Research, 11, 43657-43661.

Almeida, C. B.; Casotti, C. A. \& Sena, E. L. S., (2018). Reflexões sobre a complexidade de um estilo de vida saudável. Avances en Enfermería, 36(2), 220229. 
Almeida, C. B. et al. (2019). Reflexão sobre o controle do acesso de quilombolas à saúde pública brasileira. Avances em Enfermería, $37(1), 92$.

Almeida, I. L. S. et al. (2020). Estilo de vida, morbidades e multimorbidade em Quilombolas adultos. ABCS Health Sciences, $45,1-7$.

Barroso, S. M.; Melo, A. P. S. \& Guimarães, M. D. C. (2014). Depressão em comunidades quilombolas no Brasil: triagem e fatores associados. Revista Panamericana de Saúde Pública, 35(4), 256-63.

Bezerra, V. M. et al. (2013). Comunidades quilombolas de Vitória da Conquista, Bahia, Brasil: hipertensão arterial e fatores associados. Caderno de Saúde Pública, 29(9), 1889-1902.

Bezerra, V. M. et al. (2015). Domínios de atividade física em comunidades quilombolas do sudoeste da Bahia, Brasil: estudo de base populacional. Cadernos de Saúde Pública, 31(6), 1213-24.

Bezerra, V. M. et al. (2014). Inquérito de Saúde em Comunidades Quilombolas de Vitória da Conquista, Bahia, Brasil (Projeto COMQUISTA): aspectos metodológicos e análise descritiva. Ciência e Saúde Coletiva, 19(6), 1835-1847.

Bonsmann, S. S. G. \& Wills, J. M. (2012). Nutrition Labeling to Prevent Obesity: Reviewing the Evidence from Europe. Current Obesity Reports, 1(3), 13440 .

Both, J. et al. (2008). Validação da escala “Perfil do Estilo de Vida Individual”. Revista Brasileira de Atividade Física Saúde, $13(1)$, 5-14.

Brasil. (2012). Cartilha do censo 2010 - Pessoas com deficiência.

Brasil. (2015). Guia de Políticas públicas para comunidades Quilombolas.

Brasil. (2008). Ministério da Saúde. Diretrizes e recomendações para o cuidado integral de doenças crônicas não-transmissíveis. Brasília.

Calais, S. L.; Andrade, L. M. B. \& Lipp, M. E. N. (2003) Diferenças de sexo e escolaridade na manifestação de Stress em adultos jovens. Psicologia: Reflexão e Crítica, 16(2), 257-263.

Cardoso, L. G. V.; Melo, A. P. S. \& Cesar, C. C. (2015). Prevalência do consumo moderado e excessivo de álcool e fatores associados entre residentes de Comunidades Quilombolas de Vitória da Conquista, Bahia, Brasil. Ciência e Saúde Coletiva, 20(3), 809-20.

Carvalho, E. Q., \& Cavalcanti, R. J. de S. (2020). Inclusão na Educação Profissional e Tecnológica: abordagem emancipatória do trabalho como princípio educativo. Research, Society and Development, 9(5), e115953219.

Clarke, P. \& Latham, K. (2014). Life course health and socioeconomic profiles of Americans aging with disability. Disability Health Journal, 7(supp.1), s15s23.

Claro, R. M. et al. (2015). Consumo de alimentos não saudáveis relacionados a doenças crônicas não transmissíveis no Brasil: Pesquisa Nacional de Saúde, 2013. Epidemiologia e Serviços Saúde, 24(2), 257-265.

Cruz, D. M. C. et al. (2015). O trabalho e a tecnologia assistiva na perspectiva de pessoas com deficiência física. Revista de Terapia Ocupacional da Universidade de São Paulo, 26(3), 382-389.

Danaei, G. et al. (2009). The Preventable Causes of Death in the United States: Comparative Risk Assessment of Dietary, Lifestyle, and Metabolic Risk Factors. PLoS Medicine, 6(4), e1000058.

Dawes, T. P. \& Coutinho, A. C. M. S. (2021). A inclusão escolar do aluno surdo: proposta bilíngue no contexto da diversidade e inclusão. Cenas Educacionais, 4, e11740.

Farias, N. \& Buchalla, C. M. (2005). A classificação internacional de funcionalidade, incapacidade e saúde da organização mundial da saúde: conceitos, usos e perspectivas. Revista Brasileira de Epidemiologia, 8(2), 187-93.

Faro, A. \& Pereira, M. E. (2011). Raça, racismo e saúde: a desigualdade social da distribuição do estresse. Estudos de Psicologia, 16(3), 271-278.

Franco, R. B., \& Oliveira Neto, A. M. de. (2020). Um Panorama Histórico do Processo de Inclusão das Pessoas com Deficiência no Mercado de Trabalho. Research, Society and Development, 9(1), e155911871.

Fundação Cultural Palmares. (2016). Instituição pública voltada para promoção e preservação da arte e da cultura afro-brasileira. [Internet]. [cited 2016 Oct 18]. Available from: http://www.palmares.gov.br/

Gomes, A. B. et al. (2020). Transtorno do espectro autista e hábito alimentar de crianças e adolescentes. Research, Society and Development, 9(10), e7319108978.

Gomes, K. O. et al. (2013). Utilização de serviços de saúde por população quilombola do Sudoeste da Bahia, Brasil. Cadernos de Saúde Pública, 29(9), 18291842 .

Green, L. W.; Kreuter, M. W. \& Green, L. W. (2005). Health program planning: an educational and ecological approach. New York: McGraw-Hill.

Hall, L.; Colantonio, A. \& Yoshida, K. (2003). Barriers to nutrition as a health promotion practice for women with disabilities. International Journal of Rehabilitation Research, 26(3), 245-247.

Holanda, C. M. A. et al. (2015). Support networks and people with physical disabilities: social inclusion and access to health services. Ciência e Saúde Coletiva, 20(1), 175-84. 
Holmbäck, I. et al. (2010). A high eating frequency is associated with an overall healthy lifestyle in middle-aged men and women and reduced likelihood of general and central obesity in men. British Journal of Nutrition, 104(7), 1065-1073.

Kawachi, I. \& Berkman, L. F. (2001). Social Ties and Mental Health. Journal Urban Health, 78(3), 458-467.

Lehnhard, G. R.; Manta, S. W. \& Palma, L. E. (2012). A prática de atividade física na história de vida de pessoas com deficiência física. Revista de Educação Física da UEM, 23(1), 45-56.

Llewellyn, G. \& Hindmarsh, G. (2015). Parents with Intellectual Disability in a Population Context. Current Developmental Disorders Reports, 2(2), 119-126.

Malta, D. C. et al. (2015). Estilos de vida da população brasileira: resultados da Pesquisa Nacional de Saúde, 2013. Epidemiol e Serviços Saúde, 24(2), 217-26.

Malta, D. C. et al. (2016). Prevalência autorreferida de deficiência no Brasil, segundo a Pesquisa Nacional de Saúde, 2013. Ciência \& Saúde Coletiva, 21(10):3253-3264.

Mateus, M. D. et al. (2008). The mental health system in Brazil: Policies and future challenges. International Journal of Mental Health Systems, 2(12), 1-8.

Mielke, G. I. et al. (2015). Prática de atividade física e hábito de assistir à televisão entre adultos no Brasil: Pesquisa Nacional de Saúde 2013. Epidemiologia e Serviços de Saúde, 24(2), 277-86.

Mussi, R. F. F. et al. (2015). Aividades Físicas Praticadas no Tempo Livre em Comunidade Quilombola do Alto Sertão Baiano. Licere, 18(1), $157-87$.

Mussi, R. F. F. et al. (2020). Inquérito de Saúde em População Quilombola Baiana: relato de uma experiência em pesquisa epidemiológica. Revista Saúde e Pesquisa, 13(675-685).

Mussi, R. F. F.; Rocha, S. V. \& Alves, T. C. (2019). Transtornos Mentais Comuns em Quilombolas Baianos, Nordeste Brasileiro. Psicologia, Saúde \& Doenças, 20(698-710).

Nogueira, G. C. et al. (2016). Perfil das pessoas com deficiência física e Políticas Públicas: a distância entre intenções e gestos. Ciência e Saúde Coletiva, 21(10), 3131-3142.

Nordenfelt, L. (2003). Action theory, disability and ICF. Journal Disability and Rehabilitation, 25(18), $1075-1079$.

Oliveira, K. M. J. et al. (2021). Atividades de Lazer em Adultos Quilombolas de uma Região Baiana. Licere, 24(3), $251-268$.

Oliveira Neto, V. E. de, \& Melo, Á. F. de. (2020). O direito das pessoas com deficiência sensorial à inclusão educacional no Supremo Tribunal Federal. Research, Society and Development, 9(2), e19921806.

Organização Mundial de Saúde. (1997). Clasificación internacional de las deficiencias, actividades e participación: un manual de las dimensiones de la inhabilitación y su funcionamiento. Genebra.

Organização Mundial de Saúde. (2010). Recomendaciones mundiales sobre la actividad física para la salud. Genebra.

Organização Pan-Americana da Saúde. (2009). Prevenção de doenças Crônicas um investimento vital. Genebra.

Pereira, F. S. \& Freitas, J. F. F. de. (2021). Atividade física e transtorno do espectro autista: uma revisão de periódicos brasileiros. Cenas Educacionais, 4 , e11933.

Pereira, R. N. \& Mussi, R. F. F. (2020). Acesso e utilização dos serviços de saúde da população negra quilombola: uma análise bibliográfica. Odeere, 5, 280303.

Quaioti, T. C. B. \& Almeida, S. S. (2006). Determinantes psicobiológicos do comportamento alimentar: uma ênfase em fatores ambientais que contribuem para a obesidade. Psicologia USP, 17(4), 193-211.

Rebouças, C. B. A. et al. (2011). Pessoa com deficiência física e sensorial: Percepção de alunos da graduação em enfermagem. ACTA Paulista de Enfermagem, 24(1), 80-86.

Rodrigues, D. N. et al. (2020a). Determinantes sociodemográficos associados ao nível de atividade física de quilombolas baianos, inquérito de 2016*. Epidemiologia d Servicos de Saúde, 29, e2018511.

Rodrigues, D. N. et al. (2020b). Determinantes sociodemográficos da Falta de Prontidão para Atividade Física em Adultos Quilombolas. Revista de Ciências Médicas e Biológicas, 19, 89-94.

Schenker, M. \& Minayo, M. C. D. S. (2004). A importância da família no tratamento do uso abusivo de drogas: uma revisão da literatura. Cadernos de Saude Publica, 20(3), 649-659.

Silva, R. N. P.; Mussi, R. F. F. \& Rocha, R. M. (2020). Acesso e utilização dos serviços de saúde por quilombolas contemporâneos baianos. Revista da Associação Brasileira de Pesquisadores/as Negros/as, 12, 449-469.

Souza Júnior, M. de; Correia, F. L. de S. \& de Queiroz, Z. F. (2021). O uso das tic na inclusão sócio laborativa da pessoa com transtorno do espectro autista e/ou outras deficiências. Cenas Educacionais, 4, e10232.

Tomey, K. M. et al. (2005). Dietary intake and nutritional status of urban community-dwelling men with paraplegia. Archives of Physical Medicine and Rehabilitation, 86(4), 664-671. 
Research, Society and Development, v. 10, n. 14, e564101422135, 2021

(CC BY 4.0) | ISSN 2525-3409 | DOI: http://dx.doi.org/10.33448/rsd-v10i14.22135

Vasconcellos, M. S. \& Sena, V. S. (2021). Três dimensões da acessibilidade para uma efetiva inclusão educacional de crianças com deficiência. Cenas Educacionais, 4, e10804.

Yokota, R. T. C. et al. (2016). Contribution of chronic conditions to gender disparities in disability in the older population in Brazil, 2013. International Journal of Public Health, 61(9), 1003-1012.

Weil, E. et al. (2002). Obesity among adults with disabling conditions. Jama, 288(10), 1265-1268. 